\title{
Potential use of the undersampling technique in the acquisition of nuclear magnetic resonance signals
}

\author{
Pablo Pérez ${ }^{\mathrm{a}}$, Andrés Santos ${ }^{\mathrm{a}, *}$, Juan José Vaquero ${ }^{\mathrm{b}, \mathrm{c}}$ \\ a E.T S.I. Telecomunicación, Ciudad Universitaria, Universidad Politécnica de Madrid, E-28040 Madrid, Spain \\ ${ }^{\mathrm{b}}$ Nuclear Medicine Department, National Institutes of Health. Bethesda, MD, USA \\ ${ }^{\mathrm{c}}$ Unidad de Medicina y Cirugía Experimental, Hospital GU Gregorio Marañón, Madrid, Spain
}

\begin{abstract}
This communication reviews the use of undersampling techniques to acquire NMR signals. Undersampling transforms bandpass free induction decay (FID) signals, centered at high frequencies, into lowpass signals or bandpass signals centered at much lower frequencies. Consequently, the analog electronic stages that perform the demodulation can be eliminated, gaining in stability and reducing the phase distortion while maintaining an equivalent or better signal to noise ratio when an adequate sampling rate is chosen. The technique has been tested on a BRUKER BIOSPEC BMT 47/40, and the results show that undersampling could be used to process NMR and MRI signals, extending the range of applications of the 'digital radio' techniques to NMR and MRI apparatus.
\end{abstract}

Keywords: Undersampling; Direct digital receiver; NMR and MRI systems; Folding back noise; NMR signal acquisition; MRI signal processing

\section{Introduction}

In a typical nuclear magnetic resonance system, the signal obtained in the receiver coil is amplified using tuned amplifiers, processed with attenuators, demodulators and intermediate frequency (IF) amplifiers, and finally shifted to zero center frequency by means of a quadrature phase sensitive detector. The last step produces two free induction decay (FID) signals, which must be lowpass filtered to remove the high-frequency components [1]. This classic architecture has been very well studied and it has to be carefully designed in order to minimize noise, channel imbalances and phase distortion [1-3].

Some of those analog sections can be replaced by digital techniques in an attempt to introduce flexibility and stability over time, and therefore an increase in the quality of the signals could be expected. One of these digital techniques is oversampling, which has been used in direct digital receivers for low field MRI or Over- hauser Imaging [4]. However, the application of this technique to high field NMR and MRI systems is limited by the minimum sampling frequency of the analog to digital converter (ADC) required [5]; such a high sampling rate can only be achieved with high-end (and consequently, expensive) ADCs. A second drawback of these high rate conversion systems is that they require the use of very fast digital electronics and large storage capacities, or the use of specialized circuits such a digital down converters [6].

As an alternative, this work presents the use of undersampling or passband sampling techniques, initially proposed by Pérez et al. [7] and Green et al. [8], which have been successfully applied in electron paramagnetic resonance detection [9], in radio communications, in what has been called 'software radio' [10], and in nuclear magnetic resonance signal processing [11]. This last reference is a particular case of the more general theory described in this work.

In the software radio case an analog to digital converter takes the radio frequency (RF) or the IF signal and samples it at a rate below the Nyquist frequency. This sampling process causes the replication of the signal spectrum at several frequency bands including 


\section{PROBE}

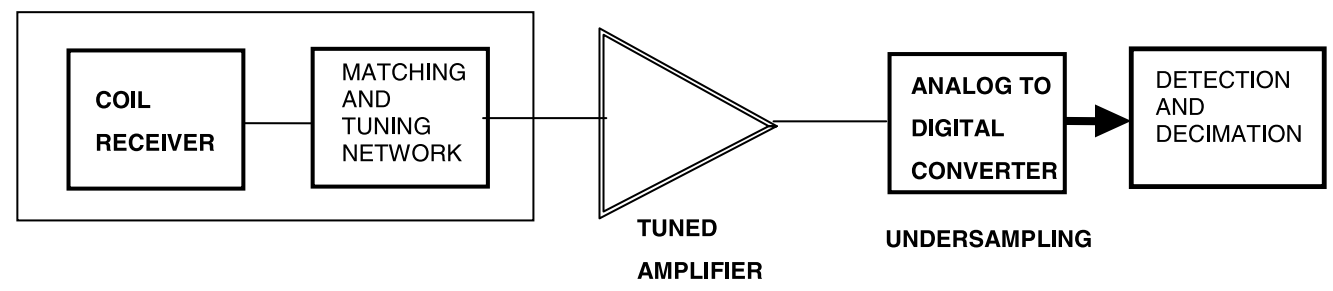

Fig. 1. Block diagram of the proposed receiver. After the tuned amplifier, the signal is down converted with undersampling. The last block (detection and decimation) is used only if the demodulated signal is not centered at zero frequency.

baseband and low frequency passband. Therefore, undersampling can be used to move the spectrum to a lower frequency (demodulate), functioning like a mixer. The original RF signal information can be completely recovered from those sampled values if the signal is actually a bandpass signal and the process of sampling satisfies the bandpass sampling theorem $[12,13]$. Then, the undersampling procedure directly applied to the amplified NMR or MRI signal as shown in Fig. 1, reduces noticeably speed and storage requirements on the data acquisition system when compared to oversampling techniques, and therefore the costs of equipment.

In order to manage all the frequency components contained in the original signal the sample and hold circuit (a first stage in the analog to digital conversion process) has to have the same bandwidth characteristics when oversampling or undersampling is used [14]. This requirement can be accomplished with recently developed wide bandwidth analog-to-digital converters, which have an input stage bandwidth that is greater than the nominal sampling rate $[15,16]$. Finally, when using undersampling techniques it is also essential to consider the noise from the aliased bands, as well as the effect of quantization.

In this work bandpass sampling applied to NMR and MRI signals in the RF band is presented and analyzed, evaluating the advantages and disadvantages of this technique. Section 2 introduces briefly the theory bases of undersampling, Section 3 presents the experiments carried out to confirm the predictions, while the results are shown and discussed in Section 4. Finally, conclusions are drawn in section $\mathrm{V}$.

\section{Theory}

Bandpass signals are characterized by having no frequency components above a frequency $f_{\mathrm{h}}$ and below a frequency $f_{1}$ (signal spectral components in the range $f_{1} \leq|f| \leq f_{\mathrm{h}}$ ) As shown in different works $[12,17]$, the aliasing produced during the sampling process can be used advantageously when sampling passband signals: a sampling rate smaller than $2 f_{\mathrm{h}}$ can be used, according to the bandpass sampling theorem for uniform and instantaneous sampling. This theorem states that a bandpass signal can be reproduced from its sample values if the sampling frequency $f_{\mathrm{s}}$ satisfies [12]

$\frac{2 f_{\mathrm{h}}}{n} \leq f_{\mathrm{s}} \leq \frac{2 f_{1}}{(n-1)}$

where $n$ is an integer that fulfills

$2 \leq n \leq \frac{f_{\mathrm{h}}}{\left(f_{\mathrm{h}}-f_{1}\right)}$

and

$\left[f_{\mathrm{h}}-f_{1}\right] \leq f_{1}$

The minimum rate, $f_{\mathrm{s}}=2\left(f_{\mathrm{h}}-f_{1}\right)$, is of interest from a theoretical point of view, but for practical applications this sampling rate can only be applied if there is no signal component at the frequencies $f_{\mathrm{h}}$ or $f_{\mathrm{l}}$. As in practical situations this cannot be guaranteed, the sampling frequency must be higher or the signal bandwidth has to be increased with a guard-band [17].

To illustrate the effect of sampling a bandpass signal with a sampling frequency that satisfies the constraints established in the above theorem an example is given in Fig. 2 [12]. In this figure, a bandpass time-domain signal $h(t)$ and its spectrum $H(f)$ are shown on the left. The center frequency of the signal is $10 f_{0}$ and the bandwidth is $2 f_{0}$. Direct application of the Nyquist theorem imposes a sampling frequency of $22 f_{0}$, but since under those conditions the undersampling theorem can be applied, a sampling frequency of $8 f_{0}(n=3)$ will produce replicas of the original spectra centered at $\pm 2 f_{0}$ and $\pm 10 f_{0}$; a low-pass filter with bandwidth $3 f_{0}$ can reconstruct the original signal $h(t)$ centered at $2 f_{0}$. From there, a numerical demodulation can be easily performed to move the filtered signal to baseband.

As shown in Fig. 2, the undersampled signal represents a copy of the original analog signal spectrum centered at a lower frequency, which in general is different from zero frequency, and sampled with a frequency greater than twice its bandwidth $\left(2 f_{0}\right)$. Therefore, in order to recover the signal at baseband, it is necessary to realize a process of digital detection (multiplication by an exponential), which permits to translate 
the copy of the spectrum to zero center frequency. A decimation stage follows this process to reduce the number of samples (detection and decimation block shown in Fig. 1).

\subsection{Noise considerations}

Other characteristics of the ADCs that become important when they are used beyond the Nyquist rate or with undersampling have been studied in the literature [13]; one of the most relevant is the degradation of the signal to noise ratio (SNR). Even under ideal conditions (meaning among other things that the signal has been perfectly band-pass filtered before the conversion stage), there is a quantization error whose spectrum extends well beyond the Nyquist frequency $f_{\mathrm{s}}\left(f_{\mathrm{s}}=2 B\right.$, being $B$ the signal bandwidth). When a quantized signal is sampled, the entire quantization noise spectrum is folded back into the baseband (aliasing), and it is reasonable to take the total quantization noise power as a measure of the noise to be expected in the baseband $[13,18]$. For sinusoidal inputs, an expression for the maximum theoretical signal to quantization noise ratio at the Nyquist rate can be derived following certain assumptions about the noise and the input signal $[19,20]$ :

$\mathrm{SNR}=6.02 N+1.76(\mathrm{~dB})$

where $N$ is the number of bits of the ADC; note that for each extra bit of resolution in the ADC, there is about $6 \mathrm{~dB}$ improvement in the SNR.

In the case of undersampling applied to relocate a bandpass signal, the noise from all the aliased bands is combined into the band where the signal of interest is relocated. As in any sampled system, the periodicity of the spectrum causes that this wideband noise appears combined into each of the $f_{\mathrm{s}} / 2$ bands. Even with an ideal antialiasing filter, the SNR is not preserved when bandpass sampling since the noise from the aliased spectra will be always overlapped into the signal.

A first estimation of the degradation of the SNR can be obtained considering an equivalent flat analog noise spectrum, ideal filters and an infinitesimally small sampling aperture. In a system with a signal with spectral

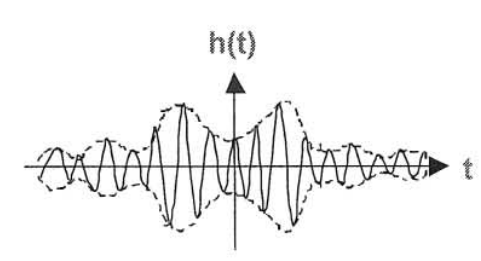

(a)

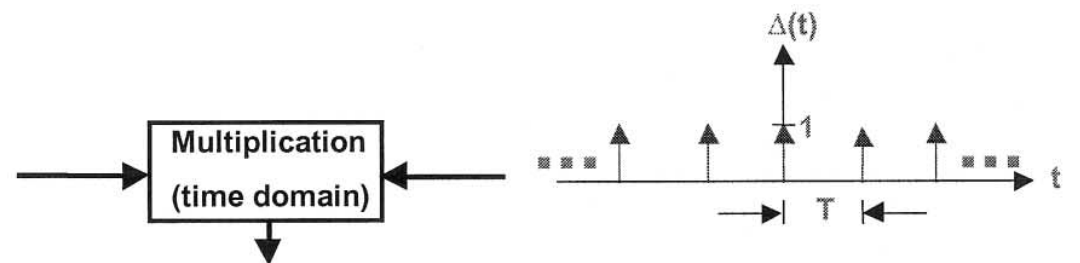

(b)

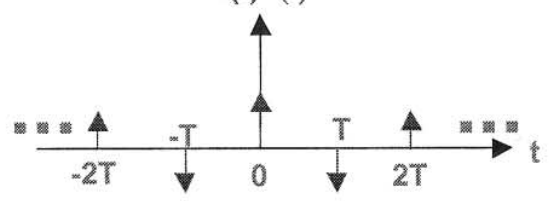

(c)

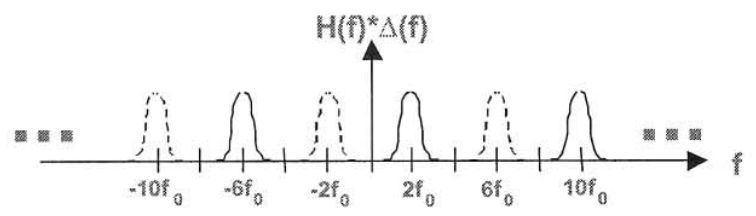

(d)

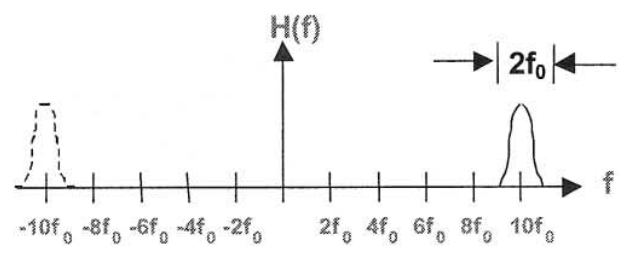

(e)

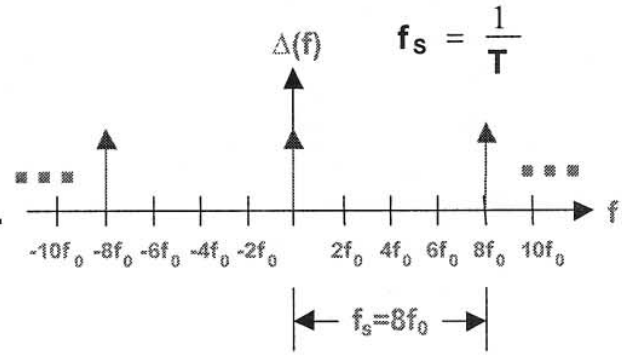

(f)

Fig. 2. Waveform and spectra of a bandpass signal sampled at less than twice the highest frequency component. (a) Bandpass time-domain signal. (b) Time-domain sampling function. (c) Time-domain sampled signal. (d) Spectrum of the sampled signal. (e) Spectrum of the passband signal. (f) Spectrum of the sampling function. 
density $S$, in-band noise power density $\sigma_{\mathrm{i}}$, and out-ofband noise power density $\sigma_{\mathrm{o}}$, the SNR for the sampled signal becomes degraded at least by the noise aliased from the bands between $d c$ and the passband, and can be estimated as [17]

$\mathrm{SNR} \approx \frac{S}{\sigma_{\mathrm{i}}+\left(n_{\mathrm{p}}-1\right) \sigma_{\mathrm{o}}}$

where $n_{\mathrm{p}}$ is the largest integer that fulfills Eq. (2).

If $\sigma_{\mathrm{i}} \approx \sigma_{\mathrm{o}}$ and $n_{\mathrm{p}} \gg 1$, then the SNR degradation $\left(D_{\mathrm{SNR}}\right)$ in decibels can be approximated by

$D_{\mathrm{SNR}} \approx 10 \log \left(n_{\mathrm{p}}\right)$

If the noise spectrum is not uniform, then the $D_{\mathrm{SNR}}$ is given by

$D_{\mathrm{SNR}} \approx 10 \log \left(\frac{B_{\mathrm{EA}}}{f_{\mathrm{s}} / 2}\right)$

where $f_{\mathrm{s}}$ is the sampling rate and $B_{\mathrm{EA}}$ is the equivalent noise bandwidth of the analog signal.

When the noise level obtained with a certain sampling frequency is unacceptable, it can be reduced by using a higher sampling frequency or larger bandguard, i.e. a smaller value of $n_{\mathrm{p}}$. After this higher frequency (but still below Nyquist) sampling, the signal spectrum can be digitally translated to baseband and decimated [21].

\subsection{Application of undersampling techniques to NMR systems}

As it was presented in the previous section, undersampling degrades the signal-to-noise ratio, but the traditional analog detector introduces some degradation as well [8], specially the analog phase-detector. According to the literature [1,2,22], a standard phasequadrature detector has a noise figure of at least $10 \mathrm{~dB}$. Then, from Eq. (7) it can be deduced that by choosing the adequate sampling frequency, undersampling can provide a digitized signal with at least an equivalent (if not better) signal-to-noise ratio than that obtained with an analog phase-quadrature detector followed by a Nyquist or oversampling analog-to-digital conversion, that is the standard procedure used in NMR and MRI systems. We are assuming that both systems use the same signal source (pre-amplifier), whose noise figure will predominate in the total noise figure of the complete chain [3], although our analysis starts at the pre-amplifier output. Here is an example: Let's assume that an analog-to-digital converter is working at the Nyquist sampling frequency $f_{\mathrm{N}}$. If this converter does not introduce any additional degradation of the signalto-noise ratio, its equivalent noise bandwidth is half of the Nyquist sampling frequency, $B_{\mathrm{EA}}=f_{\mathrm{N}} / 2$. According to Eq. (7), to obtain a degradation smaller than or equal to $10 \mathrm{~dB}$, the sampling frequency has to be $f_{\mathrm{s}} \geq f_{\mathrm{N}} / 10$. This means that in the case of the MRI system used in the experiments, whose Larmor frequency was $200 \mathrm{MHz}$ (Nyquist sampling frequency: 400 Msamples/s), the use of the undersampling technique with a sampling frequency of 40 Msamples/s (undersampling by a factor of 10) will affect the final SNR in a way similar to the conventional analog phase-quadrature detector plus Nyquist analog-to-digital conversion.

It is also necessary to remark that when applying digital techniques to acquire the NMR RF signal, the coherence of the excitation pulses applied to the sample during excitation is important. The oscillating RF field produced by the transmitter coil when a sequence of pulses is applied at times $t_{1}, t_{2}, \ldots, t_{k}$ with duration $\tau_{1}, \tau_{2}, \ldots, \tau_{k}$ can be represented by the function

$H(t)=\sum_{k} A_{k}(t) \cos \left(w t+\varphi_{k}\right)$

where $A_{k}(t)=0$ outside the interval $t_{k} \leq t \leq t_{k}+\tau_{k}$. Those pulses are called incoherent if the phases $\varphi_{k}$ are randomly distributed, and coherent if their values can be controlled (in particular if they have the same value $\varphi$ ). These variations in phase values certainly affect the final phase of the received signal, specially the encoding phase in a 2 DFT image reconstruction process. If coherent pulses are used, the receiver structure presented in Fig. 1 is still valid, since in this case the phase of the received signal is not modified from line to line in the scanning process. However, when the NMR equipment uses incoherent pulses, it is necessary either to have a reference signal or to implement a method to compensate the phase fluctuations. When processing the echoes with the goal of reconstructing an image, it is necessary to acquire either the reference signal used by the demodulation stage in the NMR equipment, or the excitation pulse itself in order to estimate the initial phase. Then, the system structure in Fig. 1 has to be modified to include an additional stage to acquire the excitation pulses in synchronism with the acquisition of the echoes. It is also possible to process the FID without any additional reference signal by using the method proposed by Chen [23] and Callaghan [24].

\section{Materials and methods}

To evaluate if undersampling is a viable alternative for processing the NMR and MRI signals, in our experiments we used a BIOSPEC BMT 47/40 MR system (BRUKER, 4.7 T, $200 \mathrm{MHz}$ for ${ }^{1} \mathrm{H}$ ), which uses incoherent excitation pulses. This is an analog system all the way down to the AD conversion of the baseband signal.

Signals were acquired with a digital oscilloscope (Tektronix TDS-524A with GPIB interface), that has an analog bandwidth limited to $500 \mathrm{MHz}$ per channel, 
sampling rates up to 500 Msamples/s, and storage capacity up to 50000 samples per channel. Data were transferred to a PC type computer via the GPIB interface after acquisition. This scope has two important limitations for this experiment: first, it has 8 bits per sample resolution, which limits the maximum theoretical SNR for every scale of the oscilloscope to 49.92 $\mathrm{dB}$ - Eq. (4) - due to the quantization noise. Second, the sampling frequency was set to predetermined values limiting the possibilities of experimenting with more adequate sampling rates.

The NMR signal (200.36 MHz center frequency) was acquired at the output of the second RF amplification stage from the scanner; depending on the conditions of operation (sequence and FOV) the bandwidth was 50 , 25 or $12.5 \mathrm{kHz}$. For quality control purposes, we also acquired the signal at the output of the analog receiver just before the AD converters, where the quadrature components of the echo signal are available in baseband $(0-100 \mathrm{KHz})$.

A $49.5 \mathrm{~mm}$ diameter spherical phantom made of glass and filled with water was used for the experiments. Echo signals were generated using a spin-echo sequence $(\mathrm{TR}=1500 \mathrm{~ms}, \mathrm{TE}=6 \mathrm{~ms}, \mathrm{FOV}=60 \times 60$ $\mathrm{mm}$, slice thickness $=5 \mathrm{~mm}$ ). The acquired data were sent to computer via GPIB interface during the TR interval, where they were stored and processed off line. The acquisitions were synchronized with a pulse generated by the scanner that signals the reading interval of the pulse sequence.

To verify the experimental results predicted by the theory, a harmonic generation effect was simulated using the MATLAB environment (The MathWorks, Natick, MA).

\subsection{FID signals acquisition}

At the RF tap point, FID signals were acquired at 2.5 Msamples/s (undersampling) while after the analog mixer the sampling rate was 0.5 Msamples/s (oversampling). The number of FID signals collected at each point was 128 and each signal was acquired during $16.384 \mathrm{~ms}$.

The undersampled signals were processed as follows:

1. Carrier frequency detection, by locating the position of the maximum in the magnitude spectrum.

2. Baseband shifting, by multiplying each signal by a complex exponential

$\exp \left(\frac{2 \pi f_{\mathrm{c}} n}{f_{\mathrm{s}}}+\varphi_{\mathrm{i}}\right)$

where $f_{\mathrm{c}}$ is the carrier frequency of the signal, $f_{s}$ is the sampling rate and $\varphi_{\mathrm{i}}$ is the phase value that permits to obtain a perfect absorption spectrum of the FID signal $[23,24]$.
3. Decimation: 512 complex values were kept for the quadrature components of each signal to match the MRI system data size. To eliminate the ringing effect introduced by the decimation filter at the ends of the record, the number of samples is reduced to 500 by removing the first six and the last six samples of each record.

4. Spectrum magnitude and phase computation: using a complex FFT, rows of two $128 \times 500$ matrices (magnitude and phase) are computed and stored.

5. Signal-to-noise ratio calculation: the mean and standard deviation of the magnitudes $\left(\mu_{\mathrm{mi}}, \sigma_{\mathrm{mi}}\right)$ and phases $\left(\mu_{\mathrm{pi}}, \sigma_{\mathrm{pi}}\right)$ were obtained point by point. The signal-to-noise ratio is estimated as follows [25]:

$\mathrm{SNR} \approx 20 \log \left(\frac{\operatorname{mean}\left(\mu_{\mathrm{mi}}\right)}{\operatorname{mean}\left(\sigma_{\mathrm{mi}}\right)}\right)$

where mean $\left(\mu_{\mathrm{mi}}\right)$ is the average value of the mean of the magnitudes across spectra and mean $\left(\sigma_{m i}\right)$ is the average of the standard deviation of the same magnitudes (magnitudes for the same frequency of the 128 spectra).

The signals acquired after the analog mixer (oversampled) were processed following the last three steps only.

\subsection{Acquisition of MR echoes in an image acquisition}

Images were acquired with a resolution of $128 \times 128$. The signals corresponding to the central line of the $\mathrm{K}$-space were acquired at the RF tap point undersampled at different rates $(0.5,1,2.5$, and 5 Msamples/s). In all cases, each signal was acquired during an interval of time of $2.3 \mathrm{~ms}$ and its acquisition started with the lead edge of the read pulse. For each sampling rate, 128 signals were acquired, the squared magnitude of the spectrum calculated, and averaged point by point in order to evaluate the effect on the SNR of different sampling rates. The signal-to-noise floor ratio was calculated using:

$\mathrm{SNR}_{\text {floor }}=10 \log \left(\frac{\overline{S^{2}}}{\overline{N_{\text {floor }}^{2}}}\right)$

where $\overline{S^{2}}$ is the average power of the components of the signal coming from the sphere and $\overline{N_{\text {floor }}^{2}}$ is the average power of the noise floor excluding spurious or harmonic components. This procedure is based on that given by Jenq for measuring noise floor and distortion of an AD converter when a sine wave is digitized [26].

To determine whether undersampling affects the phase information, one of the signals acquired at 2.5 Msamples/s was demodulated in quadrature (with a constant phase factor to compensate that introduced by the carrier of the excitation pulse in the NMR system) and compared with the signals obtained from the corresponding analog detector. 

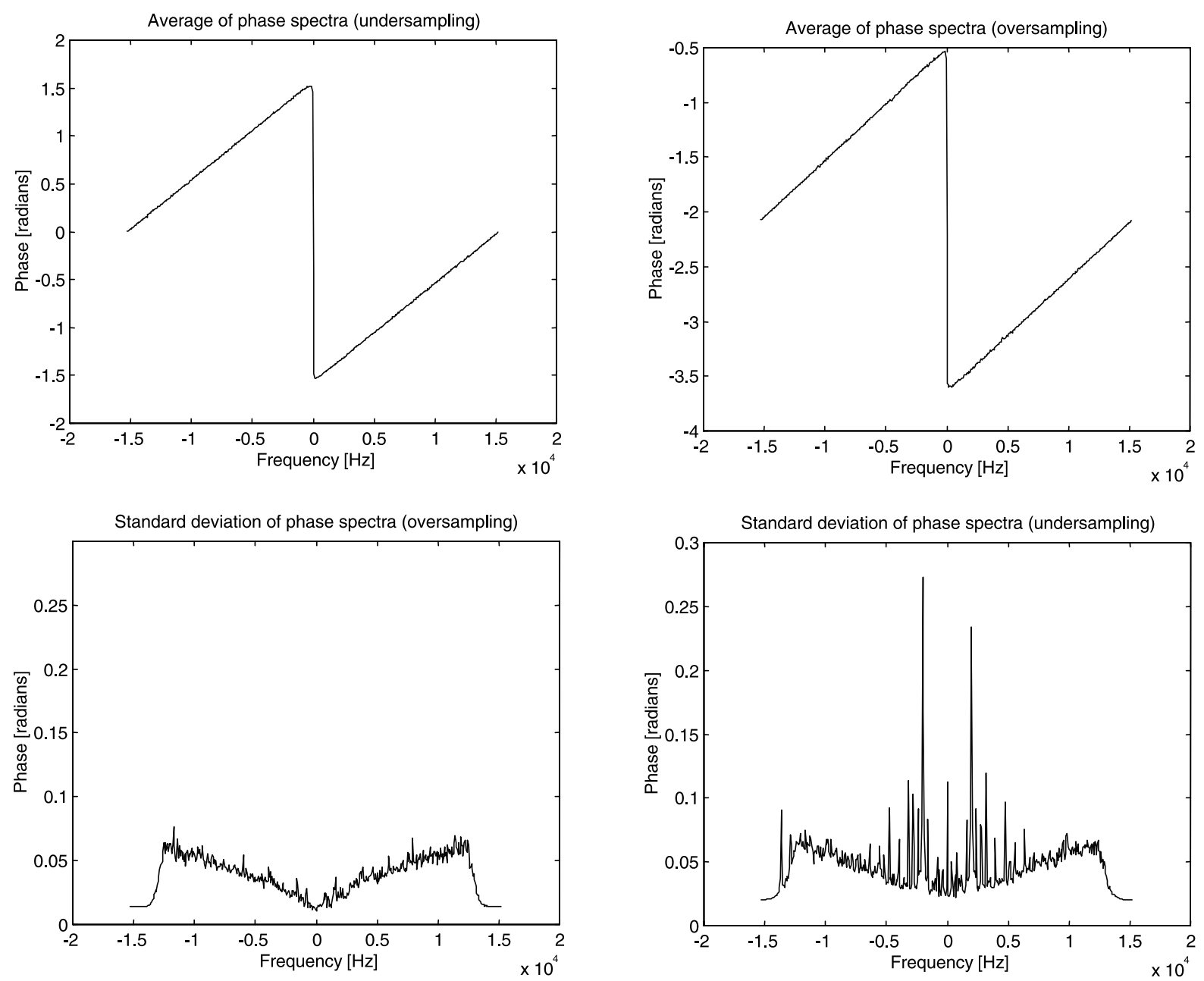

Fig. 3. Average values (top) and S.D. (bottom) of 128 phase spectra from FID signals acquired after the quadrature receiver using oversampling (left), and before the analog demodulation stage using undersampling (right).

\section{Results and discussion}

The results of the FID signals acquisition are presented in Figs. 3 and 4; in both cases (under and oversampling), the mean value of the phase follows a linear function, covering the same range of values, $\pi$ radians (Fig. 3, panels a and b). The difference between undersampling and oversampling is the presence of a displacement, which is due to the different phase of the reference signal used in the detection processes. Panels $\mathrm{c}$ and $\mathrm{d}$ of the same figure depict the standard deviation of the phase values. The standard deviation in both cases follows similar curves but the one obtained from the undersampled data presents larger deviations in some discrete frequency values. This difference in spurious components is an artifact of the sampling process due to the high correlation of the quantization error with the input signal [27], as happens when working with sinusoidal inputs [28]; it is due to a small number of quantization levels and a big distance be- tween levels (violation of the Bennett conditions [29]). Besides, the phase fluctuations of the carrier of the FID signal, that is also quantized, introduce fluctuations in the phases of these spurious components and therefore cause the large changes in the standard deviation of phase spectrum.

Additionally, and due to the oscilloscope ADC characteristics, it was necessary to truncate the FID signal. This time-domain truncation introduces additional frequency-domain components (leakage) because of the side-lobe characteristics of the sinc function and the incoherent acquisition of the signal [30]. This harmonic generation effect has been perfectly reproduced by the computer simulations, and the observed results in the analysis of the standard deviations of magnitude spectra in the simulated conditions are similar to Fig. 4, panel $\mathrm{d}$, validating the hypothesis about their origin: In the simulations the distortion due to the spurious components disappeared when using 12-bit quantization. These results let us conclude that a more adequate 
ADC scheme in the experiments (more bits per sample and no FID truncation) will reduce, if not completely remove, this artifact.

SNR for both cases have been estimated using Eq. (9). When undersampling the RF signal at 2.5 Msamples/s the SNR was $27.5 \mathrm{~dB}$, while when oversampling the baseband signal this value was $37.4 \mathrm{~dB}$. This apparently lower performance of the undersampling technique is in our opinion caused by the very low sampling rate used in the experiment since it was not possible to select the adequate rate. We try to prove our point with the experiment described in the next paragraph. It is also possible to improve the undersampling SNR by using three different mechanisms: (1) a higher sampling rate, according to Eq. (7); (2) a higher number of quantization levels, to reduce the correlation between the signal and the quantization noise; and (3) avoiding the necessity of FID truncation and thus eliminating the spurious components shown in Figs. 3 and 4.
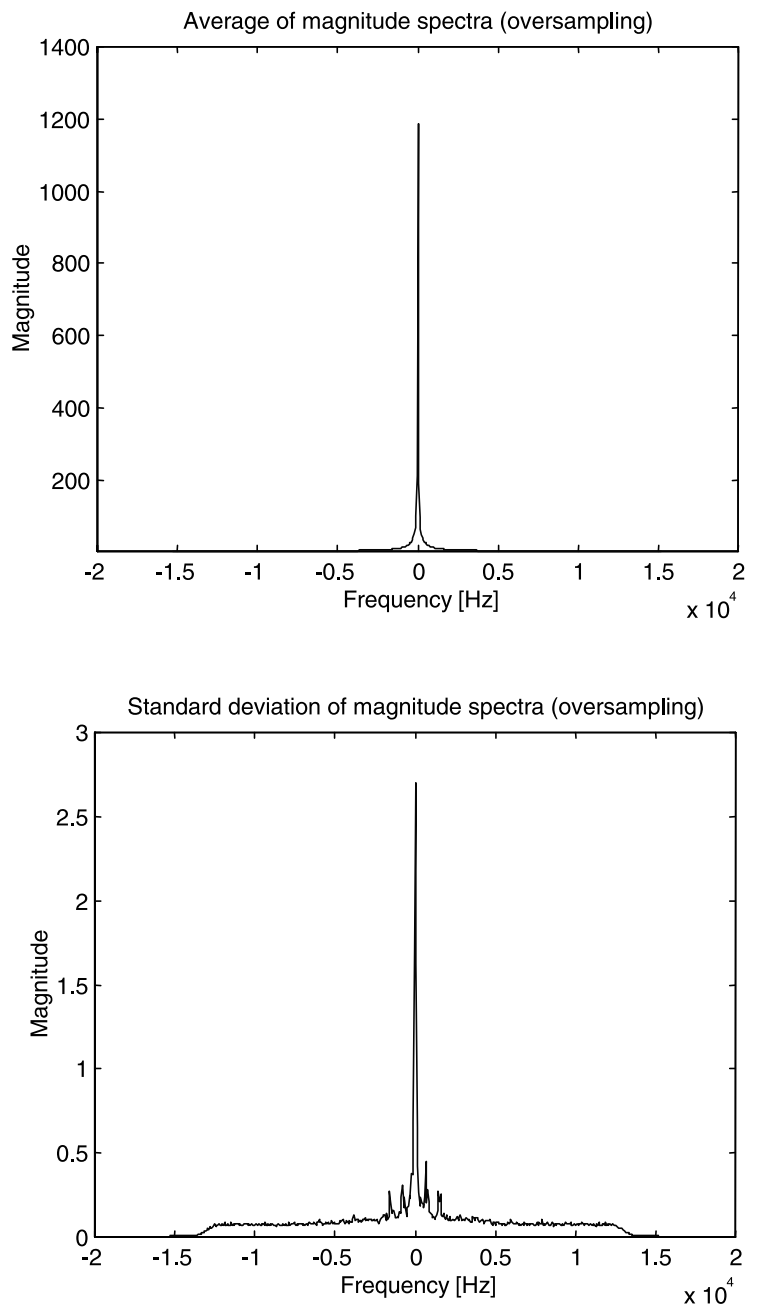

Signal to floor noise ratios presented in Table 1 were obtained analyzing the spectra of the signals corresponding to the central line of the K-space of the uniform spherical sample acquired with different sampling rates, using Eq. (10). The last two columns show the relative decrease in SNR introduced by too low sampling rates compared with the 5 Msamples/s reference case. Measured results and values predicted by Eq (7) are almost identical.

Fig. 5 shows the central line of the K-space in the image acquisitions. The quadrature components obtained with undersampling are shown in panel a, while panel $b$ shows the components obtained by oversampling (a constant phase correction was introduced in the undersampled results). These results shown that, except by a scale factor of approximately $1 / 224$ and a higher noise level (due to a too low sampling rate, as explained above), undersampling and oversampling produce the same profiles. This means that undersam-
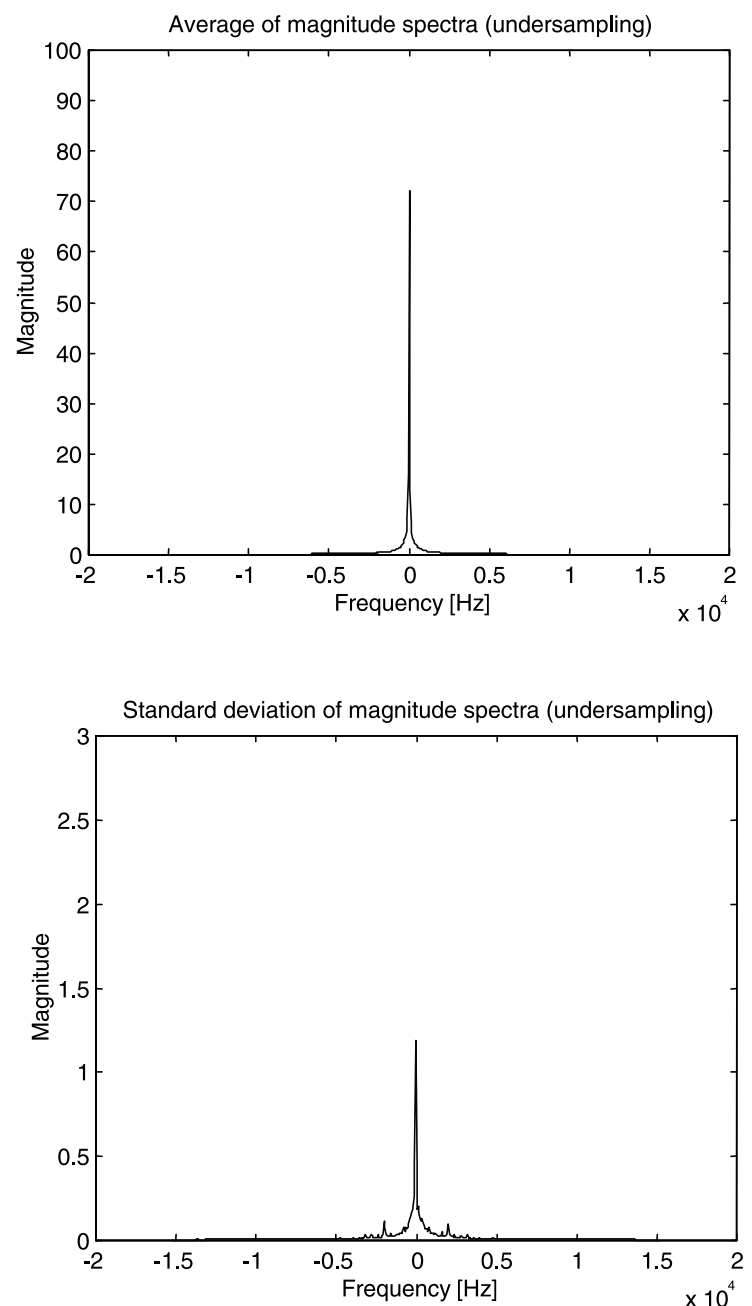

Fig. 4. Averages (top) and standard deviations (bottom) of 128 magnitude spectra from FID signals acquired after the quadrature receiver with oversampling (left), and before the analog demodulation stage using undersampling (right). 
Table 1

Dependency of the signal to noise floor ratio with the sampling rate

\begin{tabular}{lccc}
\hline Sampling rate $($ Msamples/s) & Measured SNR floor $_{(\mathrm{dB})}$ & Relative $^{\mathrm{a}}$ measured $\mathrm{SNR}_{\text {floor }}(\mathrm{dB})$ & Relative $^{\mathrm{a}}$ predicted SNR \\
\hline 0.5 & 29.2 & -10.2 & -10.0 \\
1 & 32.4 & -7.0 & -7.0 \\
2.5 & 36.4 & -3.0 & -3.0 \\
5 & 39.4 & - & - \\
\hline
\end{tabular}

Comparison of theoretical predictions and experimental results.

${ }^{a}$ Relative to a sampling rate of 5 Msamples/s.
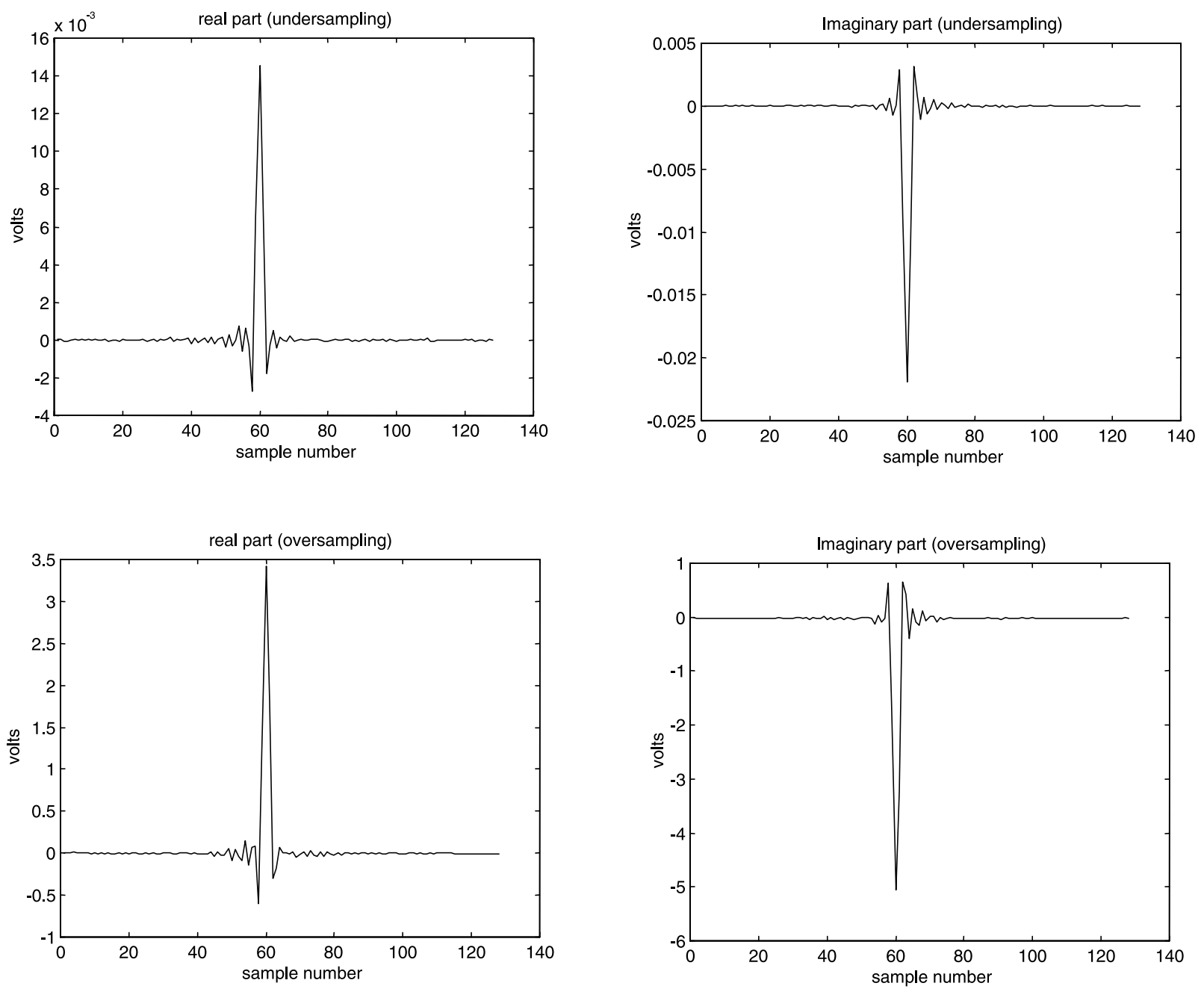

Fig. 5. Real (left) and imaginary (right) parts obtained from the echo corresponding to the central line of the K-space of a spherical phantom. (a) Components obtained before the analog demodulation stage using undersampling at 5 Msamples/s. (b) Components obtained after the quadrature receiver using oversampling at $2.5 \mathrm{Msamples} / \mathrm{s}$.

pling can be used in MRI experiments since it does not modify the relative phase of the components of the nuclear magnetic resonance signal and, if the phase relationship between lines of the K-space is preserved, the undersampled reconstructed image will not differ from the oversampled one.

\section{Conclusions}

This work presents undersampling as an alternative way of processing NMR and MRI signals, instead of the traditional analog processing with analog phasequadrature detectors. The theory predicts that an ade- 
quate selection of the sampling rate and quantization levels may actually produce better SNR than the analog alternative. The experimental results allowed us to characterize the SNR deterioration due to the folding of the aliased spectra caused by the undersampling technique: This degradation varied depending on the parameter selection, according to the theoretical predictions. It was also demonstrated that using the undersampling technique, the information coded in frequency and phase is correctly recovered, making feasible the use of this technique for MRI. Additional advantages are that this technique overcomes some of typical problems of analog processing, like low frequency noise, dc offsets, phase errors or channel imbalances in the quadrature detection. NMR systems that work with incoherent excitation pulses require phase compensation methods, like for example the one proposed in this work. With the advent of recent ADCs with wider bandwidth for the sample and hold stage, the undersampling technique may be useful in systems with higher field magnets, opening its use to a varied range of new applications.

\section{Acknowledgements}

The authors thank the Magnetic Resonance Unit of the Instituto Pluridisciplinar (UCM) for giving us access to use their NMR system, specially to Dr Jesús Ruiz-Cabello, Palmira Villa and Marien Fernández for their help with the setup of our experiments, and Professor Francisco del Pozo (UPM) for the electronic equipment provided. P. Pérez also thanks the Instituto de Cooperación con Iberoamérica and DGAPA, UNAM, that have support his postgraduate fellowship.

\section{References}

[1] Chen C-N, Hoult DI. Biomedical Magnetic Resonance Technology. Bristol: Adam Hilger, 1989.

[2] Rhode UL, Whitaker J, Bucher TTN. Communications Receivers: Principles and Design, 2nd Edition. New York: McGrawHill, 1997.

[3] Vlaardingerbroek MT, den Boer JA. Magnetic Resonance Imaging. Berlin: Spinger-Verlag, 1996.

[4] Luyten MJ, Korbee D, Claasen-Vujcic T, Mehlkopf AF. Programmable direct receiver for low field magnetic resonance imaging. Proc. of SMR/ESMRMB, 1995, p. 934.

[5] Halámek J, Kasal M, Vondra V, Villa M, Cofrancesco P. Direct digital receiver for NMR and MRI. Proc. of SMR/ESMRMB, 1996, p. 276.
[6] Harris Semiconductor, Digital signal processing. Databook. Harris Semiconductor Literature Department, 1994.

[7] Pérez P, Santos A, Vaquero JJ, Rivera M, del Pozo F. Reducing analog circuitry by sampling NMR signals at low speeds. Magn Reson Mat Phys Biol Med (MAG*MA) 1996;IV(II):100.

[8] Green DP, Balcom BJ. Undersampling the NMR signal. Poster: hardware 031, Experimental Nuclear Magnetic Resonance Conference, Asilomar, CA, 1998.

[9] Hyde JS, Mchaourab HS, Camenisch TG, Ratke JJ, Cox RW, Froncisz W. Electron paramagnetic resonance detection by timelocked subsampling. Rev Sci Instrum 1998;69(7):2622-8.

[10] Analog Devices Inc. Software radios. Microwave J. 1996;39:128-136.

[11] Stormont RS, Anas MC, Pelc NJ. Radio frequency receiver for a NMR instrument, US patent number 4,992,736, 1989.

[12] Brigham EO. The Fast Fourier Transform and its Application. New Jersey: Prentice Hall, 1988.

[13] Wepman JA. Analog-to-digital converters and their applications in radio receivers. IEEE Commun Mag 1995;33:39-45.

[14] Munroe SC, Lu AK. 2- $\mu \mathrm{m}, 1.6-\mathrm{mW}$ gated- $\mathrm{g}_{\mathrm{m}}$ sampler with 72-dB SFDR for $\mathrm{f}_{\mathrm{s}}=160 \mathrm{Ms} / \mathrm{s}$ and $\mathrm{f}_{\mathrm{in}}=320.25 \mathrm{MHz}$. IEEE J. Solid-State Circuits 1998;33:400-409.

[15] Analog Devices Inc. 14-bit 80 Msps IF-sampling Analog-to-digital converter. http://www.analog.com/products/descriptions/ AD6644.html, 1999.

[16] National Semiconductor. CLC5958 14-bit, 52 Msps A/D converter. http://www.national.com, 1999.

[17] Vaughan RG, Scott NL, White DR. The theory of bandpass sampling. IEEE Trans Signal Process 1991;39:1973-84.

[18] Cattermole KW. Principles of Pulse Code Modulation. London: Iliffe Books, 1969.

[19] Ifeachor EC, Jervis BW. Digital Signal Processing, A Practical Approach, 1st Edition. New York: Addison-Wesley, 1993.

[20] Aziz PM, Sorensen HV, Der Spiegel JV. An overview of sigmadelta converters. IEEE Signal Process Mag 1996;13:61-84.

[21] Crochiere RE, Rabiner LR. Multirate Digital Signal Processing. New Jersey: Prentice Hall, 1983.

[22] Microwave Communications Laboratories, Inc. Single sideband quadrature modulators/demodulators. http://mcli.com/ quadratu.html, 1997.

[23] Chen C, Khan L. An iterative phase correction program for nuclear magnetic resonance (NMR) spectra. Comp Methods Prog Biomed 1988;26:81-4.

[24] Callaghan P. Principles of Nuclear Magnetic Resonance Microscopy, Reprinted. Oxford: Claredon Press, 1995.

[25] Gonzalez RC, Woods RE. Digital Image Processing, 3rd Edition. Massachusetts: Addison Wesley, 1992.

[26] Jenq YC. Measuring harmonic distortion and noise floor of an A/D converter using spectral averaging. IEEE Trans Instrum Meas 1988;37:525-8.

[27] Gray RM. Quantization noise spectra. IEEE Trans Inform Theory 1990;36:1220-44.

[28] Claasen TACM, Jongepier A. Model for the Power Spectral Density of quantization noise. IEEE Trans Acoust Speech, Signal Processing 1981;ASSP-29:914-917.

[29] Gray RM, Stockham TG. Dithered quantizers. IEEE Trans Inform Theory 1993;39:805-12.

[30] Kester W. High speed design techniques. Analog Devices, http:// www.analog.com, 1996 